\title{
Study of Klebsiella pneumoniae strains resistant to carbapenems isolated from blood in eastern Liguria
}

\author{
Giulia Carnesecchi, Silvia Reali \\ ASL 4 Chiavarese, Via Don Bobbio 23, Lavagna (Ge)
}

Key words: Carbapenem resistance, Blood, K. pneumoniae

Isolamento da emocolture di Klebsiella pneumoniae resistente ai carbapenemi in un'area del levante ligure

\section{SUMMARY}

Objectives. Study of multi-resistant Klebsiella pneumoniae strains isolated from blood cultures collected from in-patients of hospitals located in eastern Liguria, and evaluation of the susceptibility to carbapenems and other antibiotics by E-test and automated methods.

Methods. At the Laboratory of Clinical Microbiology, of Lavagna Hospital in eastern Liguria, 397 Klebsiella pneumoniae strains were collected from in-patients from different wards of hospitals sites, during the year $20 \mathrm{II}$. They included II 5 isolates from blood cultures (aerobic and anaerobic) and various biological materials.

All strains were tested in the laboratory for their susceptibility to antibiotics.

Results. Of the II 5 strains of Klebsiella pneumoniae collected from blood cultures $59.1 \%$ showed resistance to imipenem, ertapenem, meropenem.

Conclusions. The data show a high incidence of resistance to carbapenems in Klebsiella pneumoniae isolated from blood cultures. This is important to implement surveillance programs for control and prevention, but also reduce the intake of antibiotics when they are not strictly necessary.

\section{INTRODUZIONE}

Le Enterobacteriaceae sono considerate tra i più comuni agenti patogeni umani, poiché possiedono la propensione a diffondersi facilmente nei diversi ambienti, contaminano spesso cibo e acqua così come diversi distretti del corpo umano e sono in grado di acquisire materiale genetico attraverso il trasferimento genico orizzontale, mediati principalmente da plasmidi e trasposoni (6).

Klebsiella pneumoniae è uno dei più importanti rappresentanti di questa famiglia di batteri e del genere Klebsiella essendo quella più spesso associata a patologie dell'uomo. È spesso la causa di infezioni dell'apparato respiratorio come polmoniti e broncopolmoniti, di infezioni del tratto urinario (soprattutto nei pazienti cateterizzati), di infezioni cutanee (ferite, ulcere, decubiti), batteremia nel sangue, setticemie e meningiti (4).

La terapia delle infezioni sostenute da K. pneumoniae si avvale di antibiotici come le ureidopenicilline protette da inibitore suicida di ß-lattamasi, cefalosporine di $2^{\circ}$ e $3^{\circ}$ generazione, monobattamici, carbapenemici e fluorochinoloni.

Il trattamento dipende dalle condizioni di salute del paziente, dalla storia medica e dalla gravità dell'infezione. La terapia deve essere sempre accompagnata da antibiogramma specie se il ceppo è di isolamento nosocomiale poiché questo ambiente è facilmente caratterizzato da resistenza multipla agli antibiotici.

I microrganismi patogeni infatti modificano il loro metabolismo in seguito a condizioni di stress, adattandosi al nuovo ambiente per sopravvivere; sviluppano così meccanismi in grado di neutralizzare o distruggere gli antibiotici che essi stessi producono.

Tra i fenomeni di antibiotico resistenza considerati emergenti a livello mondiale e sottoposti a sorveglianza troviamo la produzione di carbapenemasi da parte degli Enterobatteri. Questi enzimi agiscono idrolizzando i farmaci carbapenemi, entrati in uso clinico nel 1985.

Conseguenze di tali resistenze sono che il paziente infetto è soggetto ad una ospedalizzazione prolungata, ha un più elevato rischio di morte e riceve antibiotici più costosi e potenzialmente più tossici (5). La mortalità attribuibile alle infezioni da Enterobacteriaceae resistenti ai carbapenemi è infatti elevata, pari al 20-30\%, potendo arrivare fino al 70\% nelle batteriemie (3).

La diffusione clonale di tali microrganismi si sviluppa con estrema facilità e parte di questa è anche associata ai trasferimenti dei pazienti nei vari ospedali (10), per questo vi è la necessità di adottare comportamenti assistenziali atti a ridurre quanto più possibile la trasmissione.

\section{Corresponding author: Silvia Reali}

ASL 4 chiavarese, Laboratorio di Microbiologia,

Via Don Bobbio 25 - 16033 Lavagna - Tel.: 0185329615 - Fax: 0185329697

E-mail: sreali@asl4.liguria.it 
Inoltre per contrastare la diffusione delle CRE (Carbapenems resistant Enterobacteria) sono state messe a punto linee guida da numerose istituzioni internazionali (Centers for Disease Control, Health Protection Agency, European Centre for Disease Prevention and Control) (13). In laboratorio i ceppi produttori di carbapenemasi sono difficili da valutare sulla base dei saggi di sensibilità agli antibiotici di routine, sia con sistemi automatici sia con sistemi manuali, in quanto la resistenza non mostra spesso un fenotipo ben definito.

Questo studio è stato condotto presso il Laboratorio di Analisi Chimico - Cliniche e Microbiologiche, settore di Microbiologia dell'Azienda Ospedaliera $\mathrm{N}^{\circ} 4$ Chiavarese con sede a Lavagna.

Tale indagine è basata sull'analisi e sulla valutazione dal punto di vista laboratoristico del fenomeno di antibiotico-resistenza dei ceppi batterici di isolamento clinico. Lo scopo è quindi quello di mostrare il percorso necessario per una corretta identificazione fenotipica di specie batteriche Gram-negative, in particolar modo di Klebsiella pneumoniae presente in emocolture, e di valutarne la sensibilità agli antibiotici carbapenemi tramite test automatizzati ed E-test.

\section{MATERIALI E METODI}

Presso il Laboratorio di Microbiologia dell'Ospedale di Lavagna nell'anno 2011 sono stati identificati in pazienti provenienti da diversi reparti del levante ligure 397 ceppi di Klebsiella pneumoniae, di cui 115 isolate da emocolture (aerobie e anaerobie) e le restanti da materiali biologici vari.

Tutti i ceppi derivanti da emocolture sono stati analizzati per la loro sensibilità agli antibiotici. I campioni di sangue sono pervenuti in laboratorio all'interno di flaconi per emocoltura FAN: (Fattore di Neutralizzazione degli Antibiotici) contiene carbone attivato che garantisce una migliore capacità di recupero dei microrganismi nei pazienti sottoposti ad antibioticoterapia (poiché in grado di catturare le molecole antibiotiche presenti nel sangue del paziente) (1).

Il sistema di incubazione e rilevazione utilizzato per le emocolture è BacT/ALERT 3D (bioMèrieux), in cui i microrganismi eventualmente presenti nel campione metabolizzano i substrati nel terreno di coltura producendo anidride carbonica.

L'accumulo di $\mathrm{CO}_{2}$ viene paragonato col livello iniziale di $\mathrm{CO}_{2}$ nel flacone e, in caso di aumento, il campione viene giudicato positivo (11).

L'identificazione batterica e la determinazione dell'antibiogramma sono state effettuate tramite il sistema automatizzato Vitek 2, che li esegue parallelamente utilizzando card differenti.
Per l'identificazione dei microrganismi Gram negativi le card sono ID-GN. I processi di inoculazione, incubazione e lettura sono completamente controllati e guidati dallo strumento e dal software del computer.

Per la valutazione della sensibilità agli antibiotici sono state utilizzate le card AST-GN24 (9), le quali contengono nei pozzetti aliquote a varie concentrazioni di antibiotici diversi.

Lo strumento esegue 4 letture per ogni pozzetto e viene monitorizzata la crescita batterica ad intervalli di 15 minuti in presenza delle diverse concentrazioni.

I dati ottenuti sono paragonati con il pozzetto di controllo e per ogni antibiotico uno specifico algoritmo converte i dati in un valore di MIC (8). La MIC è la minima concentrazione inibente, ovvero la concentrazione più bassa di antibiotico in grado di inibire la crescita di un determinato organismo (espressa in $\mu \mathrm{g} / \mathrm{ml}$ ).

Viene applicata per modulare le terapie nella scelta del farmaco, nel suo dosaggio, nel monitoraggio delle resistenze e per prevedere l'effetto terapeutico di un antibiotico.

Gli isolati batterici vengono quindi classificati in sensibili, intermedi o resistenti a seconda che la MIC sia maggiore o minore a determinati punti di interruzione (14).

La sensibilità agli antibiotici viene confermata eseguendo l'E-test.

È un metodo quantitativo di secondo livello per la determinazione della MIC, utilizzato quando i metodi di routine di laboratorio forniscono risultati dubbi o quando si devono ricercare resistenze specifiche o confermare.

Consiste in una sottile striscia di plastica, in cui su un lato è riportata la scala di lettura graduata, sull'altro è immobilizzato un gradiente di concentrazioni predefinite di antibiotico costituito da 15 diluizioni. Secondo la procedura viene prima di tutto preparata la sospensione batterica (per i Gram negativi la densità della sospensione deve essere tra $0.5-1$ McFarland) (12).

Si immerge un tampone sterile nella sospensione da inoculare e si striscia manualmente l'intera superficie del terreno in tre direzioni, ruotando ogni volta la piastra di circa 90 gradi, al fine di assicurare l'uniforme distribuzione dell'inoculo. Si lascia asciugare e si applica la striscia di E-test. Infine si mette ad incubare per 12-24 ore in termostato. Il risultato sarà che il gradiente di antibiotico viene rilasciato sul terreno di coltura e apparirà una zona di inibizione ellittica, che andrà ad intersecare la scala di valori di MIC in cui la concentrazione di antibiotico testato inibisce la crescita del microrganismo in questione. Questo valore corrisponde alla MIC utilizzata per scegliere il 
trattamento ottimale nella cura del paziente.

\section{RISULTATI}

Sono state presi in considerazione 397 ceppi di $K$. pneumoniae provenienti da reparti del levante ligure e isolate nel corso dell'anno 2011 da campioni clinici vari (emocolture, urine, tamponi, feci, liquido pleurico ecc...).

Tutti i campioni, in seguito all'identificazione fenotipica, sono stati sottoposti a test di sensibilità agli antibiotici: alcuni ceppi sono risultati carbapenemi-resistenti, altri invece si sono mostrati sensibili verso questa classe di antibiotici.

I risultati della resistenza e sensibilità a imipenem, ertapenem e meropenem per ciascun materiale sono riportati nella tabella sottostante.

Da quanto indicato nella tabella nell'anno 2011 sono state identificate 115 Klebsiella pneumoniae derivanti da emocolture (aerobie e anaerobie). Di 115 Klebsiella il $59.1 \%$ ha mostrato di essere resistente all'azione di Imipenem, Ertapenem e Meropenem, il 40.9\% si è invece rivelato sensibile all'azione chemioterapica degli antibiotici saggiati presi qui in considerazione.

Da notare è l'elevata incidenza di antibiotico-resistenze nell'emocolture, a differenza dei 199 ceppi di $K$. pneumoniae derivanti da urine, le quali hanno mostrato per i carbapenemi una percentua- le di resistenza del $25.1 \%$ e di sensibilità del $74.9 \%$.

È quindi possibile dire che più l'infezione è localizzata in siti "nobili", che dovrebbero essere assolutamente sterili, come il torrente circolatorio e il liquido pleurico, maggiore è la possibilità di trovare batteri resistenti, difficili da trattare con terapie antibiotiche. Nella vescica e nel tratto urinario sono invece presenti germi uropatogeni (il più comune è $l^{\prime} E$. coli) con un'incidenza medio bassa del $25.1 \%$ di resistenza agli antibiotici carbapenemi.

La resistenza ai farmaci Imipenem, Ertapenem e Meropenem, per quanto riguarda il totale dei ceppi isolati dai vari materiali, risulta essere del $40.5 \%$, e questo dato è molto elevato se si pensa che l'Imipenem resta, anche a distanza di tempo dalla sua prima introduzione in terapia, il farmaco che in vitro esprime il più ampio spettro antibatterico. Nel corso dell'anno 2011, in ceppi di $K$. pneumoniae, è stata valutata anche la presenza di ß-lattamasi in grado di inibire le cefalosporine di terza generazione. I microrganismi produttori di $ß$-lattamasi ad ampio spettro vengono definiti "ESBL positivi".

Nella seguente tabella vengono mostrate le percentuali di resistenza e sensibilità a questa classe di ß-lattamici per ciascun materiale biologico:

Tabella I. Microrganismo: Klebsiella pneumoniae spp pneumoniae; materiale, numero e incidenza di resistenza e sensibilità ai carbapenemi

\begin{tabular}{|c|c|c|c|c|c|}
\hline MATERIALE & Tot. casi & $\mathbf{R}$ & $\mathbf{S}$ & $\% \mathbf{R}$ & $\% \mathbf{S}$ \\
\hline BRONCOASPIRATO & 20 & 10 & 10 & 50.0 & 50.0 \\
\hline CATETERE VENOSO- ARTERIOSO- URINARIO & 12 & 12 & 0 & 100.0 & 0 \\
\hline COLTURA ASCESSO CHIUSI AEROBI & 2 & 0 & 2 & 0 & 100.0 \\
\hline ESPETTORATO PER G.VARI & 5 & $\mathrm{I}$ & 4 & 20.0 & 80.0 \\
\hline FECI PER COLTURA & $\mathrm{I}$ & $\mathrm{I}$ & 0 & 100.0 & 0 \\
\hline LAV. BRONCOALVEOLARE DX & 4 & 2 & 2 & 50.0 & 50.0 \\
\hline LAV. BRONCOALVEOLARE SX & 2 & 2 & 0 & 100.0 & 0 \\
\hline LIQUIDO PLEURICO & 2 & 2 & 0 & 100.0 & 0 \\
\hline LIQUIDO PLEURICO ANAEROBI & $\mathrm{I}$ & I & 0 & 100.0 & 0 \\
\hline MATERIALE BIOLOGICO VARIO & 12 & 8 & 4 & 66.7 & 33.3 \\
\hline SANGUE AEROBI I CAMPIONE & 37 & 22 & 15 & 59.5 & 40.5 \\
\hline SANGUE AEROBI II CAMPIONE & 14 & 8 & 6 & 57.1 & 42.9 \\
\hline SANGUE AEROBI III CAMPIONE & 12 & 4 & 8 & 33.3 & 66.7 \\
\hline SANGUE ANAEROBI I CAMPIONE & 35 & 23 & 12 & 65.7 & 34.3 \\
\hline SANGUE ANAEROBI II CAMPIONE & 10 & 8 & 2 & 80.0 & 20.0 \\
\hline SANGUE ANAEROBI III CAMPIONE & 2 & 0 & 2 & 0 & 100.0 \\
\hline SANGUE PER COLTURA & 5 & 3 & 2 & 60.0 & 40.0 \\
\hline TAMPONE CUTANEO & 4 & 0 & 4 & 0 & 100.0 \\
\hline TAMPONE DA FERITA & 15 & 3 & 12 & 20.0 & 80.0 \\
\hline TAMPONE URETRALE & 2 & 0 & 2 & 0 & 100.0 \\
\hline TAMPONE VAGINALE & $\mathrm{I}$ & $\mathrm{I}$ & 0 & 100.0 & 0 \\
\hline URINE & 152 & 24 & 128 & 15.8 & 84.2 \\
\hline URINE DA CATETERE & 37 & 20 & 17 & 54.1 & 45.9 \\
\hline URINE DA CATETERE ESTEMPORAN. & 10 & 6 & 4 & 60.0 & 40.0 \\
\hline TOTALE & 397 & 161 & 236 & 40.5 & $\mathbf{5 9 . 5}$ \\
\hline
\end{tabular}


Tabella 2. Klebsiella pneumoniae spp pneumoniae; materiale, numero e incidenza di resistenza e sensibilità ai B-lattamici.

\begin{tabular}{|c|c|c|c|c|c|}
\hline MATERIALE & Tot. casi & $\mathbf{R}$ & $\mathbf{S}$ & $\% \mathbf{R}$ & $\% \mathbf{S}$ \\
\hline BRONCOASPIRATO & 20 & 4 & 16 & 20.0 & 80.0 \\
\hline CATETERE VENOSO- ARTERIOSO- URINARIO & 12 & 0 & 12 & 0 & 100.0 \\
\hline COLTURA ASCESSO CHIUSI AEROBI & 2 & 0 & 2 & 0 & 100.0 \\
\hline ESPETTORATO PER G.VARI & 5 & 0 & 5 & 0 & 100.0 \\
\hline FECI PER COLTURA & $\mathrm{I}$ & 0 & I & 0 & 100.0 \\
\hline LAV. BRONCOALVEOLARE DX & 4 & 0 & 4 & 0 & 100.0 \\
\hline LAV. BRONCOALVEOLARE SX & 2 & 0 & 2 & 0 & 100.0 \\
\hline LIQUIDO PLEURICO & 2 & 0 & 2 & 0 & 100.0 \\
\hline LIQUIDO PLEURICO ANAEROBI & $\mathrm{I}$ & 0 & $\mathrm{I}$ & 0 & 100.0 \\
\hline MATERIALE BIOLOGICO VARIO & 12 & 0 & 12 & 0 & 100.0 \\
\hline SANGUE AEROBI I CAMPIONE & 37 & 13.9 & 23.1 & 37.5 & 62.5 \\
\hline SANGUE AEROBI II CAMPIONE & 14 & 0 & 14 & 0 & 100.0 \\
\hline SANGUE AEROBI III CAMPIONE & 12 & 0 & 12 & 0 & 100.0 \\
\hline SANGUE ANAEROBI I CAMPIONE & 35 & 5.4 & 29.6 & 15.4 & 84.6 \\
\hline SANGUE ANAEROBI II CAMPIONE & 10 & 0 & 10 & 0 & 100.0 \\
\hline SANGUE ANAEROBI III CAMPIONE & 2 & 0 & 2 & 0 & 100.0 \\
\hline SANGUE PER COLTURA & 5 & 1.3 & 3.7 & 25.0 & 75.0 \\
\hline TAMPONE CUTANEO & 4 & 0 & 4 & 0 & 100.0 \\
\hline TAMPONE DA FERITA & 15 & 11.3 & 3.7 & 75.0 & 25.0 \\
\hline TAMPONE URETRALE & 2 & 0 & 2 & 0 & 100.0 \\
\hline TAMPONE VAGINALE & $\mathrm{I}$ & 0 & $\mathrm{I}$ & 0 & 100.0 \\
\hline URINE & 152 & 17.2 & 134.8 & 11.3 & 88.7 \\
\hline URINE DA CATETERE & 37 & 6.8 & 30.2 & 18.5 & 81.5 \\
\hline URINE DA CATETERE ESTEMPORAN. & 10 & 1.7 & 8.3 & 16.7 & 83.3 \\
\hline TOTALE & 397 & 61.6 & 335.4 & 15.5 & 84.5 \\
\hline
\end{tabular}

La percentuale totale di ceppi ESBL positivi nell'anno 2011 è risultata essere del 15.5\%, e ciò sta a dimostrare il fatto che negli ultimi anni in $K$. pneumoniae ha iniziato a circolare una resistenza mediata da ß-lattamasi a spettro esteso capaci di idrolizzare le cefalosporine più recenti.

L'incidenza di resistenza alle cefalosporine riscontrata nei ceppi derivanti da emocolture è del $33.3 \%$ e questo dato è da considerarsi rilevante visto l'elevata incidenza di resistenza mostratasi anche nei confronti dei farmaci carbapenemi.

Si è quindi di fronte a germi considerati multiresistenti, contro cui le possibilità di terapia sono limitate.

\section{CONCLUSIONI}

Klebsiella pneumoniae è responsabile di un gran numero di colonizzazioni soprattutto nei reparti "a rischio" (Terapia intensiva, Rianimazione, Terapia intensiva neonatale), e il frequente isolamento di ceppi multi-resistenti conferma l'importanza di programmi di sorveglianza per il controllo e la prevenzione.

Essenziale è l'utilizzo non eccessivo degli agenti microbici, considerati una delle principali cause che inducono i batteri a sviluppare meccanismi di resistenza per sopravvivere.

Le possibilità di terapia contro germi multi-resi- stenti sono molto limitate infatti, purtroppo, il $30 \%$ dei pazienti considerati critici ricevono trattamenti inadeguati in caso di setticemia, con il $62 \%$ di mortalità (2).

Tutt'oggi non esistono vaccini disponibili per prevenire le infezioni da parte dei produttori di carbapenemasi, quindi la sfida di microbiologi ed altri specialisti è quella di sviluppare metodi migliori per il rilevamento dei microrganismi resistenti, al fine di poter meglio controllare il dilagare di infezioni in tutti gli ambiti (8).

\section{REFERENZE}

1. AMCLI e APSI (a cura di), Traccia per la formulazione di linee guida per l'emocoltura.

2. Ibrahim EH, Sherman G, Ward S, Fraser VJ, Kollef $\mathrm{MH}$. "The influence of inadequate antimicrobial treatment of bloodstream infections on patient outcomes in the ICU setting". Chest, 2000; 118: 146-55.

3. Martinez JL, Fajardo A, Garmendia L, Hernandez A, Linares JF, Solano LM, Sánchez MB. "A global view of antibiotic resistance". FEMS Microbiology Reviews, 2008; 33.

4. Lanciotti E. Principi di microbiologia, Zanichelli, Bologna; 2008.

5. Nordmann P, Naas T. e Cuzon G. "The real threat of Klebsiella pneumoniae carbapenemase-producing bacteria". Lancet Infect Dis. 2009; 9 (4): 228-36.

6. Nordmann P, Naas T, Poirel L. "Global Spread of Carbapenemase-producing Enterobacteriaceae". Emerging Infectious Diseases. 2011; 17 (10). 
7. Hawkey PM, Jones AM. "The changing epidemiology of resistance", Journal of Antimicrobial Chemotherapy, 2009; 64: 3-10.

8. Tenover F. Atti della conferenza sull'evoluzione e il rilevamento della resistenza nei batteri, (CDC, Atlanta USA 6 e 7 giugno 1996).

9. VITEK 2, Manuale di formazione. 2006; 226 Q Vers. A

10. http://asr.regione.emiliaromagna.it/wcm/asr/aree_di_pro- gramma/rischioinfettivo/gr_ric/pr_antibres/pubblicazioni/carbapenemasi territorio/link/carbapenemasi-territorio.pdf

11. http://www.biomerieux.it/servlet/srt/bio/italy/home

12. http://www.biomerieux-diagnostics.com/etest

13. http://www.cdc.gov/drugresistance/index.html

14. http://www.eucast.org 